\title{
Towards a Semantic Gas Source Localization under Uncertainty
}

\author{
Javier Monroy, Jose-Raul Ruiz-Sarmiento, Francisco-Angel Moreno, \\ Cipriano Galindo, and Javier Gonzalez-Jimenez \\ Machine Perception and Intelligent Robotics group (MAPIR) \\ Dept. of System Engineering and Automation \\ University of Malaga. Spain.
}

\begin{abstract}
This work addresses the problem of efficiently and coher-ently locating a gas source in a domestic environment with a mobilerobot, meaningefficientlythe coverage of the shortest distance as pos-sible andcoherentlythe consideration of different gas sources explaining the gas presence. The main contribution is the exploitation, for thefirst time, of semantic relationships between the gases detected and theobjects present in the environment to face this challenging issue. Ourproposal also takes into account both the uncertainty inherent in thegas classification and object recognition processes. These uncertaintiesare combined through a probabilistic Bayesian framework to provide apriority-ordered list of (previously observed) objects to check. Moreoverthe proximity of the different candidates to the current robot locationis also considered by a cost function, which output is used for planningthe robot inspection path. We have conducted an initial demonstrationof the suitability of our gas source localization approach by simulatingthis task within domestic environments for a variable number of objects, and comparing it with an greedy approach.
\end{abstract}

Published in: Int. Conf. on Information Processing and Management of Uncertainty in KnowledgeBased Systems. Cadiz, Spain. 2018. Part of the Communications in Computer and Information Science book series (CCIS, volume 855)

DOI: http://dx.doi.org/10.1007/978-3-319-91479-4_42

More Info: http://mapir.isa.uma.es/mapirwebsite/index.php/mapir-downloads/papers/283

Cite this paper as: Monroy J., Ruiz-Sarmiento JR., Moreno FA., Galindo C., Gonzalez-Jimenez J. (2018) Towards a Semantic Gas Source Localization Under Uncertainty. In: Medina J., OjedaAciego M., Verdegay J., Perfilieva I., Bouchon-Meunier B., Yager R. (eds) Information Processing and Management of Uncertainty in Knowledge-Based Systems. Applications. IPMU 2018. Communications in Computer and Information Science, vol 855. Springer, Cham
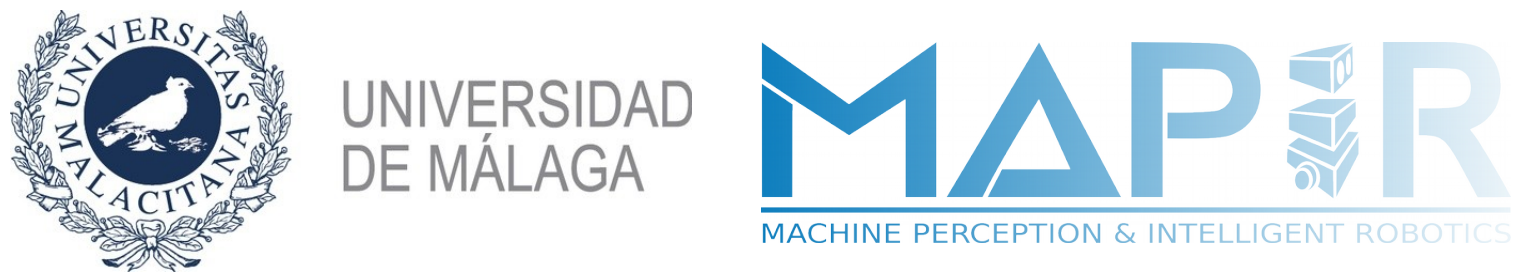